VERSITA

\author{
TATRA \\ MOUNTaiNs \\ Mathematical Publications \\ DOI: $10.2478 / \mathrm{v} 10127-011-0038-9$ \\ Tatra Mt. Math. Publ. 50 (2011), 79-86
}

\title{
THE INCLUSION-EXCLUSION PRINCIPLE WITHOUT DISTRIBUTIVITY
}

\author{
JANA KeLEMENOVÁ
}

\begin{abstract}
Inspired by the article of P. Grzegorzewski [The inclusion-exclusion principle for IF-events, Inform. Sci. 181 (2011), 536-546], who has worked two generalizations of the inclusion-exclusion principle for IF-events, a generalization of the inclusion-exclusion principle for mappings with values in semigroups is presented here. The main idea is in replacing the distributivity and idempotency laws, by one new axiom.
\end{abstract}

\section{Introduction}

P. Grzegorzewski gave two generalizations of the inclusion-exclusion principle in [3] for IF-events applying two definitions for the union of IF-events. As a reaction on this paper we have proved the principle for mappings from the set of IF-sets to the semigroup [4]. We have worked on generalizations of his theorem. First, we gave a theorem of the inclusion-exclusion principle on semigroups in [4]. The same we have made for the special case, the mapping from the set of IF-sets to the unit interval. Continuing with this topic, another method of the proof of the inclusion-exclusion principle for mappings with values in semigroups is presented in this paper. The conditions of distributivity and idempotency were replaced by the new axiom.

The classical inclusion-exclusion principle states

$$
\begin{aligned}
\mathcal{P}\left(A_{1} \cup \ldots \cup A_{n}\right)= & \sum_{i=1}^{n} \mathcal{P}\left(A_{i}\right)-\sum_{i<j}^{n} \mathcal{P}\left(A_{i} \cap A_{j}\right) \\
& +\sum_{i<j<k}^{n} \mathcal{P}\left(A_{i} \cap A_{j} \cap A_{k}\right)-\cdots+(-1)^{n+1} \mathcal{P}\left(\bigcap_{i=1}^{n} A_{i}\right)
\end{aligned}
$$

(C) 2011 Mathematical Institute, Slovak Academy of Sciences. 2010 Mathematics Subject Classification: 20 M99.

Keyw ords: semigroup, distributivity law, inclusion-exclusion principle, Gödel connectives.

This article was supported by VEGA 1/0621/11. 
for any sequence $A_{1}, \ldots, A_{n}$ from the domain of $\mathcal{P}: \mathcal{R} \rightarrow R$ (where $\mathcal{P}$ is non-negative, additive function in probability theory).

P. Grzegorzewski has defined the probability on IF-event $A$ by the formula

$$
\mathcal{P}(A)=\left[\int_{\Omega} \mu_{A} d P, 1-\int_{\Omega} \nu_{A} d P\right],
$$

where $\mathcal{P}$ is the probability measure through $\Omega$.

This inclusion-exclusion principle has the form [3]:

$$
\begin{aligned}
\mathcal{P}\left(\bigcup_{i=1}^{n} A_{i}\right)= & \sum_{i=1}^{n} \mathcal{P}\left(A_{i}\right)-\sum_{i<j} \mathcal{P}\left(A_{i} \cap A_{j}\right)+\sum_{i<j<k} \mathcal{P}\left(A_{i} \cap A_{j} \cap A_{k}\right)-\cdots \\
& \cdots+(-1)^{n+1} \mathcal{P}\left(A_{1} \cap A_{2} \cap \ldots \cap A_{n}\right) ; \\
\mathcal{P}\left(\oplus_{i=1}^{n} A_{i}\right)= & \sum_{i=1}^{n} \mathcal{P}\left(A_{i}\right)-\sum_{i<j} \mathcal{P}\left(A_{i} \odot A_{j}\right)+\sum_{i<j<k} \mathcal{P}\left(A_{i} \odot A_{j} \odot A_{k}\right)-\ldots \\
& \cdots+(-1)^{n+1} \mathcal{P}\left(A_{1} \odot A_{2} \cap \ldots \odot A_{n}\right) .
\end{aligned}
$$

Other authors (Lavinia Ciungu, Beloslav Riečan and Mária $\mathrm{K} \mathrm{u} \mathrm{k} \mathrm{ová)} \mathrm{have} \mathrm{worked} \mathrm{this} \mathrm{topic} \mathrm{on} \mathrm{IF-sets} \mathrm{and} \mathrm{IF-events} \mathrm{by} \mathrm{different} \mathrm{methods}$ and using different operators. We mention a brief summary of their results. The axiomatic definition of probability on IF-sets given in [6] is the following.

A mapping $m: \mathcal{F} \longrightarrow[0,1]$ is an IF-state if the following properties are satisfied:

1. $m\left(1_{\Omega}, 0_{\Omega}\right)=1, m\left(0_{\Omega}, 1_{\Omega}\right)=0$;

2. $m(A \oplus B)=m(A)+m(B)-m(A \odot B)$ for all $A, B \in \mathcal{F}$;

3. $A_{n} \nearrow A$ implies $m\left(A_{n}\right) \nearrow m(A)$.

Let $m: \mathcal{F} \longrightarrow[0,1]$ be an IF-state. Then there are probability measures

$$
P, Q: \mathcal{S} \longrightarrow[0,1] \text { and } \alpha \in[0,1]
$$

such that

$$
m(A)=\int_{\Omega} \mu_{A} d P+\alpha\left(1-\int_{\Omega}\left(\mu_{A}+\nu_{A}\right) d Q\right) \quad \text { for all } A \in \mathcal{F} .
$$

The result of L. C i ung u [2] using Eukasziewicz operators is following. Let $A_{i}$ be IF-events, $A_{i}=\left(\mu_{A_{i}}, \nu_{A_{i}}\right), i=1, \ldots, n$. Let $m$ be an IF-state and

$$
m(A)=\int_{\Omega} \mu_{A} d P+\alpha\left(1-\int_{\Omega}\left(\mu_{A}+\nu_{A}\right) d Q\right) .
$$


Then $m$ satisfies the inclusion-exclusion principle

$$
m\left(\bigoplus_{i=1}^{n} A_{i}\right)=\sum_{i=1}^{n} m\left(A_{i}\right)-\sum_{i<j}^{n} m\left(A_{i} \odot A_{j}\right)+\cdots+(-1)^{n+1} m\left(\bigodot_{i=1}^{n} A_{i}\right)
$$

In the proof of L. Ci ung u representation theorem from [1] was used.

Another representation theorem by $\mathrm{R}$ i eč a n and $\mathrm{C}$ i u n g u [1], corresponding to axiomatic definition of probability from $\mathrm{R}$ iečan [7] was used in the proof of M. K u k ová [5]:

Any probability $\overline{\mathcal{P}}: \mathcal{F} \rightarrow \mathcal{J}$ can be expressed by the formulas:

$$
\overline{\mathcal{P}}(A)=\left[\overline{\mathcal{P}^{b}}(A), \overline{\mathcal{P}^{\sharp}}(A)\right]
$$

and

$$
\begin{aligned}
& \overline{\mathcal{P}^{b}}(A)=\int_{X} \mu_{A} d P+\alpha \int_{X}\left(1-\mu_{A}-\nu_{A}\right) d Q, \\
& \overline{\mathcal{P}^{\sharp}}(A)=\int_{X} \mu_{A} d R+\beta \int_{X}\left(1-\mu_{A}-\nu_{A}\right) d S .
\end{aligned}
$$

K u k ová [5] proved:

$$
\begin{aligned}
\overline{\mathcal{P}}\left(\bigcup_{i=1}^{n} A_{i}\right)= & \sum_{i=1}^{n} \overline{\mathcal{P}}\left(A_{i}\right)-\sum_{i<j} \overline{\mathcal{P}}\left(A_{i} \cap A_{j}\right) \\
& +\sum_{i<j<k} \overline{\mathcal{P}}\left(A_{i} \cap A_{j} \cap A_{k}\right)-\cdots+(-1)^{n+1} \overline{\mathcal{P}}\left(A_{1} \cap A_{2} \cap \ldots \cap A_{n}\right) .
\end{aligned}
$$

K u k o vá used also the Łukasiewicz connectives:

$$
\begin{aligned}
& A \oplus B=\left(\left(\mu_{A}+\mu_{B}\right) \wedge 1,\left(\nu_{A}+\nu_{B}-1\right) \vee 0\right), \\
& A \odot B=\left(\left(\mu_{A}+\mu_{B}-1\right) \vee 0,\left(\nu_{A}+\nu_{B}\right) \wedge 1\right)
\end{aligned}
$$

to prove another form of the principle [5], that is

$$
\begin{aligned}
\overline{\mathcal{P}}\left(\bigoplus_{i=1}^{n} A_{i}\right)= & \sum_{i=1}^{n} \overline{\mathcal{P}}\left(A_{i}\right)-\sum_{i<j} \overline{\mathcal{P}}\left(A_{i} \odot A_{j}\right) \\
& +\sum_{i<j<k} \overline{\mathcal{P}}\left(A_{i} \odot A_{j} \odot A_{k}\right)-\cdots+(-1)^{n+1} \overline{\mathcal{P}}\left(A_{1} \odot A_{2} \cap \ldots \odot A_{n}\right) .
\end{aligned}
$$




\section{Inclusion-exclusion principle without distributivity for mapping with values in semigroups}

As it is mentioned in the introduction, we skip the conditions of distributivity and idempotency in the algebraic system $(\mathcal{G},+, \cdot)$, where "." is commutative binary operation, and $(\mathcal{G},+)$ is a commutative semigroup.

The only required condition is the axiom

$$
m((a+b) \cdot c)+m(a \cdot b \cdot c)=m(a \cdot c)+m(b \cdot c) .
$$

Example 2.1. Let $\mathcal{S}$ be a $\sigma$-algebra of subsets of a set $X$. Let $H$ be a linear vector Banach Riesz space. Then, any vector measure $m: \mathcal{S} \rightarrow H$ satisfies the above condition.

\section{Assumptions 2.2 .}

- $(\mathcal{G},+, \cdot)$ is an algebraic system, where $(\mathcal{G},+)$ is a commutative semigroup and "." is commutative binary operation,

- $m: \mathcal{G} \rightarrow H$ is a mapping from the algebraic system $(\mathcal{G},+, \cdot)$ to the commutative semigroup $(H,+)$, satisfying the valuation property

$$
m(a+b)+m(a \cdot b)=m(a)+m(b),
$$

- there holds the axiom

$$
m((a+b) \cdot c)+m(a \cdot b \cdot c)=m(a \cdot c)+m(b \cdot c) .
$$

TheOREM 2.3. For $n$ even

$$
\begin{aligned}
& m\left(\sum_{k=1}^{n} a_{k}\right)+\sum_{k=1}^{n / 2} \sum_{1 \leq i_{1}<i_{2}<\cdots<i_{2 k} \leq n} m\left(a_{i_{1}} \cdot a_{i_{2}} \cdots a_{i_{2 k}}\right) \\
& =\sum_{k=1}^{n / 2} \sum_{1 \leq i_{1}<i_{2}<\cdots<i_{2 k-1} \leq n} m\left(a_{i_{1}} \cdot a_{i_{2}} \cdots a_{i_{2 k-1}}\right) .
\end{aligned}
$$

For $n$ odd there holds

$$
\begin{aligned}
& m\left(\sum_{k=1}^{n} a_{k}\right)+\sum_{k=1}^{((n+1) / 2)-1} \sum_{1 \leq i_{1}<i_{2}<\cdots<i_{2 k} \leq n} m\left(a_{i_{1}} \cdot a_{i_{2}} \cdots a_{i_{2 k}}\right) \\
& =\sum_{k=1}^{(n+1) / 2} \sum_{1 \leq i_{1}<i_{2}<\cdots<i_{2 k-1} \leq n} m\left(a_{i_{1}} \cdot a_{i_{2}} \cdots a_{i_{2 k-1}}\right) .
\end{aligned}
$$

In the following examples on the sum of even and odd number of elements from $(\mathcal{G},+, \cdot)$ the process used in the proof for $n$ general is presented. We add the same terms to both sides of the equation and use the valuation property. 


\section{THE INCLUSION-EXCLUSION PRINCIPLE WITHOUT DISTRIBUTIVITY}

Example 2.4. For $n=3$. Let $a, b, c \in \mathcal{G}$. Using the valuation property (I) we can write

$$
m((a+b)+c)+m((a+b) \cdot c)=m(a+b)+m(c),
$$

from where we have:

$$
\begin{aligned}
& m(a+b+c)+m((a+b) \cdot c)+m(a \cdot b)+m(a \cdot b \cdot c) \\
& =m(a+b)+m(c)+m(a \cdot b)+m(a \cdot b \cdot c), \\
& m(a+b+c)+m(a \cdot c)+m(b \cdot c)+m(a \cdot b) \\
& =m(a+b)+m(c)+m(a \cdot b)+m(a \cdot b \cdot c), \\
& m(a+b+c)+m(a \cdot b)+m(a \cdot c)+m(b \cdot c) \\
& =m(a)+m(b)+m(c)+m(a \cdot b \cdot c) .
\end{aligned}
$$

A little bit complicated is the example for $n$ even.

Example 2.5. For $n=4$

$$
\begin{aligned}
& m((a+b+c)+d)+m((a+b+c) \cdot d) \\
& =m(a+b+c)+m(d) \\
& m(a+b+c+d)+m(a \cdot c)+m(b \cdot c)+m(a \cdot b)+m(a \cdot d+b \cdot d+c \cdot d) \\
& =m(a+b+c)+m(d)+m(a \cdot c)+m(b \cdot c)+m(a \cdot b), \\
& m(a+b+c+d)+m(a \cdot c)+m(b \cdot c)+m(a \cdot b)+m(a \cdot d+b \cdot d+c \cdot d) \\
& =m(a)+m(b)+m(c)+m(d)+m(a \cdot b \cdot c), \\
& \quad m(a+b+c+d)+m(a \cdot c)+m(b \cdot c)+m(a \cdot b)+m(a \cdot d+b \cdot d+c \cdot d) \\
& \quad+m(a \cdot d \cdot b \cdot d)+m(a \cdot d \cdot c \cdot d)+m(b \cdot d \cdot c \cdot d) \\
& =m(a)+m(b)+m(c)+m(d)+m(a \cdot b \cdot c)+m(a \cdot b \cdot d) \\
& \quad+m(a \cdot c \cdot d)+m(b \cdot c \cdot d), \\
& \quad m(a+b+c+d)+m(a \cdot c)+m(b \cdot c)+m(a \cdot b)+m(a \cdot d)+m(b \cdot d)+m(c \cdot d) \\
& \quad+m(a \cdot b \cdot c \cdot d) \\
& =m(a)+m(b)+m(c)+m(d) \\
& \quad+m(a \cdot b \cdot c)+m(a \cdot b \cdot d)+m(a \cdot c \cdot d)+m(b \cdot c \cdot d) .
\end{aligned}
$$


Proof of the Theorem 2.3. We use the method of mathematical induction here. The assumption is that the principle for $n$ elements of $(\mathcal{G},+, \cdot)$ holds and we will prove, that it holds also for $n+1$ elements.

At first, we will prove the principle for $n$ even. From the induction assumption we have

$$
\begin{aligned}
& m\left(\sum_{k=1}^{n} a_{k}\right)+\sum_{k=1}^{n / 2} \sum_{1 \leq i_{1}<i_{2}<\cdots<i_{2 k} \leq n} m\left(a_{i_{1}} \cdot a_{i_{2}} \cdots a_{i_{2 k}}\right) \\
& =\sum_{k=1}^{n / 2} \sum_{1 \leq i_{1}<i_{2}<\cdots<i_{2 k-1} \leq n} m\left(a_{i_{1}} \cdot a_{i_{2}} \cdots a_{i_{2 k-1}}\right) .
\end{aligned}
$$

The unique condition used here is the axiom (II) in its general form:

$$
\begin{aligned}
& m\left(\left(\sum_{k=1}^{n} a_{k}\right) \cdot a_{n+1}\right)+\sum_{k=1}^{n / 2} \sum_{1 \leq i_{1}<i_{2}<\cdots<i_{2 k} \leq n} m\left(a_{i_{1}} \cdot a_{i_{2}} \cdots a_{i_{2 k}} \cdot a_{n+1}\right) \\
& =\sum_{k=1}^{n / 2} \sum_{1 \leq i_{1}<i_{2}<\cdots<i_{2 k-1} \leq n} m\left(a_{i_{1}} \cdot a_{i_{2}} \cdots a_{i_{2 k-1}} \cdot a_{n+1}\right) .
\end{aligned}
$$

By the help of (I) and by adding the same terms to both sides of the equation we get

$$
\begin{aligned}
& m\left(\sum_{k=1}^{n+1} a_{k}\right)+m\left(\left(\sum_{k=1}^{n} a_{k}\right) \cdot a_{n+1}\right)+\sum_{k=1}^{n / 2} S_{2 k}^{(n)} \\
& \quad+\sum_{k=1}^{n / 2} \sum_{1 \leq i_{1}<i_{2}<\cdots<i_{2 k} \leq n} m\left(a_{i_{1}} \cdot a_{i_{2}} \cdots a_{i_{2 k}} \cdot a_{n+1}\right) \\
& =m\left(\sum_{k=1}^{n} a_{k}\right)+m\left(a_{n+1}\right) \\
& \quad+\sum_{k=1}^{n / 2} S_{2 k}^{(n)}+\sum_{k=1}^{n / 2} \sum_{1 \leq i_{1}<i_{2}<\cdots<i_{2 k} \leq n} m\left(a_{i_{1}} \cdot a_{i_{2}} \cdots a_{i_{2 k}} \cdot a_{n+1}\right) .
\end{aligned}
$$




\section{THE INCLUSION-EXCLUSION PRINCIPLE WITHOUT DISTRIBUTIVITY}

We use the induction assumption (III) on the right side of the equation

$$
\begin{aligned}
& m\left(\sum_{k=1}^{n+1} a_{k}\right)+\sum_{k=1}^{n / 2} S_{2 k}^{(n)} \\
& \quad+\sum_{k=1}^{n / 2} \sum_{1 \leq i_{1}<i_{2}<\cdots<i_{2 k} \leq n} m\left(a_{i_{1}} \cdot a_{i_{2}} \cdots a_{i_{2 k}} \cdot a_{n+1}\right)+m\left(\left(\sum_{k=1}^{n} a_{k}\right) \cdot a_{n+1}\right) \\
& =\sum_{k=1}^{n / 2} S_{2 k-1}^{(n)}+m\left(a_{n+1}\right)+\sum_{k=1}^{n / 2} \sum_{1 \leq i_{1}<i_{2}<\cdots<i_{2 k} \leq n} m\left(a_{i_{1}} \cdot a_{i_{2}} \cdots a_{i_{2 k}} \cdot a_{n+1}\right) .
\end{aligned}
$$

There can be used the result (V) for the left side of the equation

$$
\begin{aligned}
& m\left(\sum_{k=1}^{n+1} a_{k}\right)+\sum_{k=1}^{n / 2} S_{2 k}^{(n)} \\
& \quad+\sum_{k=1}^{n / 2} \sum_{1 \leq i_{1}<i_{2}<\cdots<i_{2 k-1} \leq n} m\left(a_{i_{1}} \cdot a_{i_{2}} \cdots a_{i_{2 k-1}} \cdot a_{n+1}\right) \\
& =\sum_{k=1}^{n / 2} S_{2 k-1}^{(n)}+m\left(a_{n+1}\right) \\
& \quad+\sum_{k=1}^{n / 2} \sum_{1 \leq i_{1}<i_{2}<\cdots<i_{2 k} \leq n} m\left(a_{i_{1}} \cdot a_{i_{2}} \cdots a_{i_{2 k}} \cdot a_{n+1}\right) .
\end{aligned}
$$

This yields the final formula of the inclusion-exclusion principle for $n+1$ odd number of elements from $(G,+, \cdot)$. Neither distributivity nor idempotency law were used.

$$
\begin{aligned}
& m\left(\sum_{k=1}^{n+1} a_{k}\right)+\sum_{k=1}^{((n+2) / 2)-1} \sum_{1 \leq i_{1}<i_{2}<\cdots<i_{2 k} \leq n+1} m\left(a_{i_{1}} \cdot a_{i_{2}} \cdots a_{i_{2 k}}\right) \\
& =\sum_{k=1}^{(n+2) / 2} \sum_{1 \leq i_{1}<i_{2}<\cdots<i_{2 k-1} \leq n+1} m\left(a_{i_{1}} \cdot a_{i_{2}} \cdots a_{i_{2 k-1}}\right) .
\end{aligned}
$$

The proof for $n$ odd is analogical, and concludes the proof. 


\section{JANA KELEMENOVÁ}

\section{Conclusions}

The further research could be focused on the relevance of this axiom for the family of IF-sets, and then to prove the inclusion-exclusion principle for IF-sets by the help of the axiom.

\section{REFERENCES}

[1] CIUnGU, L.-RIEČAN, B.: General form of probabilities on IF-sets, in: Proc. WILF '09, Palermo, Italy, Lecture Notes in Comput. Sci., Vol. 5571, Springer-Verlag, Berlin, 2009, pp. 101-107.

[2] CIUNGU, L.: The inclusion-exclusion principle for IF-states, Inform. Sci., 2011 (to appear).

[3] GRZEGORZEWSKI, P.: The inclusion-exclusion principle for IF-events, Inform. Sci. 181 (2011), 536-546.

[4] KELEMENOVÁ, J.: The inclusion-exclusion principle in semigroups, in: Recent Advances in Fuzzy Sets, IF-Sets, Generalized Nets and Related Topics, Vol. I, IBS PAN-SRI PAS, Warsaw, 2011, pp. 87-94.

[5] KUKOVÁ, M.: The Inclusion-Exclusion Principle for IF-events, Inform. Sci., 2011 (to appear).

[6] RIEČAN, B.: M-probability theory on IF-events, in: New Dimensions in Fuzzy Logic and Related Technologies, Proc. of 5th EUSFLAT '07, Vol. I. (M. Štěpnička, et al., eds.), Universitas Ostraviensis, Ostrava, 2007, pp. 227-230.

[7] RIEČAN, B.: A descriptive definition of the probability on intuitionistic fuzzy sets, in: Proc. EUSFLAT '03 (M. Wagenecht and R. Hampet, eds.), Zittau-Goerlitz Univ. Appl. Sci., Dordrecht, 2003, pp. 263-266. 